\title{
Synchronization to Big Data: Nudging the Navier-Stokes Equations for Data Assimilation of Turbulent Flows
}

\author{
Patricio Clark Di Leoni, ${ }^{1,2, *}$ Andrea Mazzino, ${ }^{3, \dagger}$ and Luca Biferale ${ }^{1, \$}$ \\ ${ }^{1}$ Department of Physics and INFN, University of Rome Tor Vergata, \\ Via della Ricerca Scientifica 1, 00133 Rome, Italy \\ ${ }^{2}$ Department of Mechanical Engineering, Johns Hopkins University, Baltimore, Maryland 21218, USA \\ ${ }^{3}$ Department of Civil, Chemical, and Environmental Engineering and INFN, \\ University of Genova, Genova 16145, Italy
}

(Received 14 May 2019; revised manuscript received 2 October 2019; accepted 13 December 2019; published 3 February 2020)

\begin{abstract}
Nudging is an important data assimilation technique where partial field measurements are used to control the evolution of a dynamical system and/or to reconstruct the entire phase-space configuration of the supplied flow. Here, we apply it to the canonical problem of fluid dynamics: three-dimensional homogeneous and isotropic turbulence. By doing numerical experiments we perform a systematic assessment of how well the technique reconstructs large- and small-scale features of the flow with respect to the quantity and the quality or type of data supplied to it. The types of data used are (i) field values on a fixed number of spatial locations (Eulerian nudging), (ii) Fourier coefficients of the fields on a fixed range of wave numbers (Fourier nudging), or (iii) field values along a set of moving probes inside the flow (Lagrangian nudging). We present state-of-the-art quantitative measurements of the scale-by-scale transition to synchronization and a detailed discussion of the probability distribution function of the reconstruction error, by comparing the nudged field and the truth point by point. Furthermore, we show that for more complex flow configurations, like the case of anisotropic rotating turbulence, the presence of cyclonic and anticyclonic structures leads to unexpectedly better performances of the algorithm. We discuss potential further applications of nudging to a series of applied flow configurations, including the problem of field reconstruction in thermal Rayleigh-Bénard convection and in magnetohydrodynamics, and to the determination of optimal parametrization for small-scale turbulent modeling. Our study fixes the standard requirements for future applications of nudging to complex turbulent flows.
\end{abstract}

DOI: 10.1103/PhysRevX.10.011023

Subject Areas: Fluid Dynamics, Nonlinear Dynamics

\section{INTRODUCTION}

Turbulence is the chaotic, nonlinear, and multiscale motion observed in fluids. From astrophysical and geophysical flows to engineering ones, it is a problem that surrounds us all $[1,2]$. Thus, observing, measuring, reconstructing, and then predicting the evolution of turbulent flows are highly important tasks with direct consequences for our day-to-day lives. A paradigmatic example is given by the problem of state estimation in geosciences, of particular importance to numerical weather prediction (NWP) [3]. The chaotic and multiscale nature of turbulence

\footnotetext{
patricio.clark@roma2.infn.it

andrea.mazzino@unige.it

"luca.biferale@roma2.infn.it
}

Published by the American Physical Society under the terms of the Creative Commons Attribution 4.0 International license. Further distribution of this work must maintain attribution to the author(s) and the published article's title, journal citation, and DOI. makes these tasks very difficult, as any small error in the initial conditions will make predictions diverge from the truth, and as it is not easy to access all active modes in a fluid flow. This is particularly troublesome when one considers that in a turbulent flow the number of active degrees of freedom (d.o.f.) grows with the Reynolds number as number of d.o.f. $\propto \operatorname{Re}^{9 / 4}$, with $\operatorname{Re}=U L / \nu$, given in terms of the typical rms velocity $U$, the energy containing scale $L$, and the fluid viscosity $\nu$. Data assimilation (DA) is the family of mathematical protocols used to reconstruct the states of a dynamical system, out of a series of previous partial measurements, in order to ensure that any future predictions will be as faithful as possible to what the actual physical reality will be, and has proven to be of key importance in the development of modern NWP [4-6].

Given the problem of trying to reconstruct the whole flow configuration out of some partial data, one may ask two crucial questions. The first one is about the quantity of information that one needs to collect in order to achieve a certain degree of reconstruction. The second one concerns how the quality, or type, of information affects the level of 
reconstruction that can be attained. The two main tools used in DA are based on either variational or ensembleaveraged approaches. Variational methods, best exemplified by the 4D-Var technique [7-9], rely on minimizing the distance between a simulated system's trajectory with the available data. In order to do this, the statistics of the errors are assumed to be Gaussian. Ensemble approaches work by performing Kalman filtering operations [10-12] on the probability distributions of different realizations of the state to be reconstructed. Similarly to the variational approaches, they also assume the statistics to be Gaussian. Operational implementations of either technique usually rely on linearizing the forward operator, so as to reduce the computational cost. This is known to generate problems when assimilating over long windows of time where the cumulative effect of nonlinearities becomes important [13]. Both techniques have proven useful in NWP and have also been applied to mildly turbulent channel flows [14-17], but they have never been utilized in fully developed turbulence, where the small-scale velocity statistics is intermittent with fat and non-Gaussian tails, and the system is strongly out of equilibrium and strongly nonlinear. This constitutes a big hurdle to overcome also for NWP, as new technological developments in computational and measuring tools allow weather forecast centers to reach resolutions where threedimensional turbulent convection becomes important $[18,19]$, signaling we are entering an era where nonlinear DA schemes have to be used. One possible scheme is particle filtering [20], which works like the Kalman-filterbased approaches but without employing the Gaussianity assumption. This scheme has already been tested in twodimensional barotropic flows [21] and weather models [22], showing better results than linear DA schemes, but still presenting nontrivial obstacles when scaling to highdimensional systems. New techniques based on machine learning have been applied to the flow reconstruction problem as well $[23,24]$, but only on two-dimensional flows. One important point to make about the aforementioned techniques is that they can be hard and/or very expensive to scale to three-dimensional flows. Take, for example, ensemble-based methods which require one to run several simulations; in a problem as computationally intensive as turbulence, the cost can become prohibitive.

In this paper, we propose to use nudging [25-27], a fully unbiased approach, to numerically study the problem of assimilating data into a turbulent flow which is characterized by a high- (infinite-) dimensional phase space with strong non-Gaussian and intermittent multiscale fluctuations $[2,28]$. Nudging has an old and prestigious past in DA history $[25,26]$. It consists of applying a penalty term to the right-hand side of the evolution equations that tries to minimize the distance between the evolved flow and the observations (see Fig. 1 for a sketch). In a way, nudging can be viewed as the application of a Newton relaxation feedback to fluid flows. In the context of NWP, different

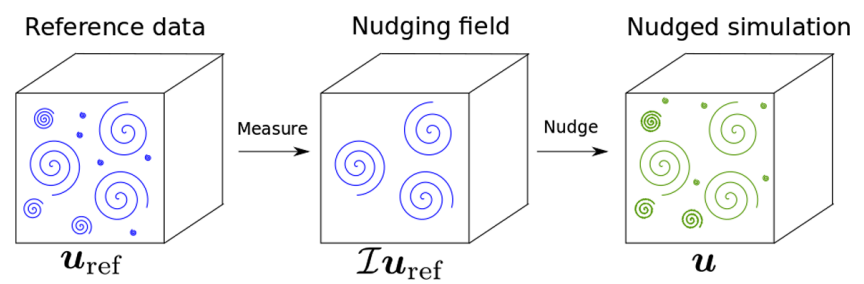

FIG. 1. Diagram outlining the nudging algorithm. In our numerical experiments the reference data come from a wellcontrolled direct numerical simulation, and the process of measuring is summarized by the filtering operation $\mathcal{I}$.

formulations of nudging have been used to study the state estimation problem using finite-dimensional dynamical systems and weather models [25,29-31], and for boundary condition matching [32-34]. In the context of turbulence, for the cases of a two-dimensional Navier-Stokes equation (NSE) [35-38], the three-dimensional Navier-Stokes $\alpha$ model [39], and Rayleigh-Bénard convection [40,41], it has been rigorously proven that given a sufficient amount of input data, a nudged field will eventually synchronize with its nudging field. Indeed, both DA [42] and nudging can be framed as a synchronization problem; see Ref. [43] for an application similar to Fourier nudging for turbulence.

Before moving on, we should note that in the current data-driven age, parameter estimation and model reconstruction are other key problems for accurate flow prediction, modeling, and control. Here the goal is to recover, out of some given data, the form and/or the parameters of the underlying partial differential equation (or ordinary differential equation) that generated such data. Modern methods include (but are not restricted to) symbolic regression coupled with sparsity methods $[44,45]$, physics informed neural networks $[46,47]$, statistical inference [48], minimum ignorance approaches [49], and even pure data assimilation methods themselves [50]. Recently, we have shown that nudging can be used to infer parameters and physics even for the case of threedimensional fully developed turbulence, both isotropic and under rotation [27]. Another related problem is the one of equation-free modeling, where recent advances haven been made in high-dimensional systems by using reservoir computing techniques [51,52], and in turbulent flows by using artificial neural networks [53]. Recent studies have gone the extra step of combining both data assimilation and machine learning for state reconstruction and model estimation [54]. All in all, these problems point to the pressing need to develop data-driven techniques that can be scaled to nonlinear and high-dimensional problems, such as turbulence, and that can both incorporate physical information when available or provide a patch (estimation) when it is not. Out of all the techniques and problems outlined, there probably is not, nor there will be, a silver bullet that can solve them all. It is the combination of suitable tools and ideas that can get the job done. 
Our novel goal is to present nudging as a tool to probe for the key d.o.f. of a flow and understand where and what we need to measure to ensure a certain level of reconstruction. We tackle problems such as if it is better to (i) place the probes in a regular equispaced way, (ii) follow measurements in a Lagrangian domain, along floating probes, or (iii) perform first a Fourier convolution to spread the information on the whole configuration space. Furthermore, we will also study nudging in the presence of inverse energy cascade [55] with the formation of highly coherent cyclonic-anticyclonic structures in rotating turbulence. These are the questions we answer here and that have not been addressed before. Many others will follow, which we leave for future research. What about bounded flows [56,57]? Is it better to place the probes close to the wall or in the bulk? What about multifield equations as in Rayleigh-Bénard [58,59] or magnetohydrodynamics (MHD) $[60,61]$ ? Can we control temperature by measuring velocity in convection, or control velocity by measuring the magnetic field in MHD? All these questions have applied and fundamental importance.

The paper is organized as follows. In Sec. II we outline how the nudging protocol works: in Sec. II A we write down the equations, in Sec. II B we give details on the numerical implementations of the technique and of the simulations performed, and in Sec. IIC we explain the different quantities we use to measure the performance of nudging. We then present the results of nudging in configuration space in Sec. III A, the results of nudging in Fourier space in Sec. III B, and the results of nudging under the presence of large-scale structures in Sec. III C. Finally, we present our conclusions in Sec. IV.

\section{METHODS}

\section{A. Nudging the Navier-Stokes equations}

Our application of nudging is based on the following protocol. Suppose we have some measurements of a reference field data $\boldsymbol{u}_{\text {ref }}$ available only on certain regions of space (or for certain Fourier modes) and with a certain cadence in time $\tau$. And suppose we know that the field evolution is described by the three-dimensional incompressible NSEs with unit density:

$$
\begin{aligned}
& \partial_{t} \boldsymbol{u}_{\text {ref }}+\boldsymbol{u}_{\text {ref }} \cdot \boldsymbol{\nabla} \boldsymbol{u}_{\text {ref }}=-\nabla p_{\text {ref }}+\nu \nabla^{2} \boldsymbol{u}_{\text {ref }}+\boldsymbol{f}_{\text {ref }}, \\
& \boldsymbol{\nabla} \cdot \boldsymbol{u}_{\text {ref }}=0, \\
& \text { + boundary conditions, }
\end{aligned}
$$

where $f_{\text {ref }}$ is a forcing mechanism and $\nu$ the viscosity. The aim is to reconstruct the whole space-time evolution of $\boldsymbol{u}_{\text {ref }}$ by evolving a numerical simulation for another incompressible velocity field $\boldsymbol{u}$, which we call the nudged field, where the distance from the input data $\boldsymbol{u}_{\text {ref }}-\boldsymbol{u}$ enters as a penalty term:

$$
\begin{aligned}
& \partial_{t} \boldsymbol{u}+\boldsymbol{u} \cdot \boldsymbol{\nabla} \boldsymbol{u}=-\nabla p+\nu \nabla^{2} \boldsymbol{u}-\alpha \mathcal{I}\left(\boldsymbol{u}-\boldsymbol{u}_{\mathrm{ref}}\right) \\
& \boldsymbol{\nabla} \cdot \boldsymbol{u}=0, \\
& \text { + boundary conditions, }
\end{aligned}
$$

where $\alpha$ is the amplitude of the nudging term and $\mathcal{I}$ is a filtering operator which projects $\boldsymbol{u}-\boldsymbol{u}_{\text {ref }}$ onto the regions of space (or the Fourier scales) in which the reference data are known. We refer to $\mathcal{I} \boldsymbol{u}_{\text {ref }}$ as the nudging field. If the cadence $\tau$ at which the observations are available does not coincide with the time step used to evolve the nudged system, one then has to define a reference field $\boldsymbol{u}_{\text {ref }}^{\tau}$ time interpolated between the two consecutive measurements. There are two very important aspects to be noted here. First, nudging can in principle be formulated for any dynamical system or partial differential equation, as done, e.g., by Refs. [62,63]; i.e., its formulation does not depend on the application to the NSE. Second, the term $\boldsymbol{f}_{\text {ref }}$ can be quite general; it does not have to be just a simple mechanical injection mechanism, it could also depend on $\boldsymbol{u}_{\text {ref }}$, for example. The filter operator $\mathcal{I}$ can take many forms, too. The first one that we address here is based on local measurements of the velocity field:

$$
\mathcal{I} \boldsymbol{u}(\boldsymbol{x}, t)=\sum_{i=1}^{N_{p}} \int \boldsymbol{u}(\boldsymbol{x}, t) \delta\left(\boldsymbol{x}-\boldsymbol{X}_{i}(t)\right) d V .
$$

Here $\boldsymbol{X}_{i}(t)$ denotes the positions of the $N_{p}$ probes where the input data are measured, and that can be fixed in space (Eulerian case) or moving with the flow (Lagrangian case) [see Eq. (8) in Sec. II B for details on the integration of Lagrangian particles]. Our implementation of Eq. (3) will actually act on small volumes and is thus a vector of the same size as $\boldsymbol{u}_{\text {ref }}$ but with zero entries outside the observing location. We present further details in Sec. II B. We refer to this scheme as "configuration space nudging." The second family of nudging protocols that we study here is based on a Fourier filtering:

$$
\mathcal{I} \boldsymbol{u}(\boldsymbol{x}, t)=\sum_{\boldsymbol{k} \in \mathcal{A}} \hat{\boldsymbol{u}}(\boldsymbol{k}, t) \exp (i \boldsymbol{k} \cdot \boldsymbol{x}),
$$

where $\hat{\boldsymbol{u}}(\boldsymbol{k})$ are the Fourier coefficients of the field $\boldsymbol{u}$ and $\mathcal{A}$ is a given subset of the Fourier space where we suppose to know the evolution of the reference field coefficients $\hat{\boldsymbol{u}}_{\text {ref }}(\boldsymbol{k})$. While in principle the set $\mathcal{A}$ can be arbitrary, in this work we always use a low-pass filter:

$$
\mathcal{A}:\left\{|\boldsymbol{k}|<k_{n}\right\}
$$

i.e., we nudge a band of large-scale modes in the flow. Simulations performed using this filter are referred to as "spectral nudging." It is very important to note that we are playing the reconstruction game in a fair way, without assuming to know anything about the external forcing mechanisms that have generated the reference field in 
Eq. (1). This is the minimal setup if we want to be realistic (in most applications even the boundary conditions are not fully under control and certainly not the space-time configuration of the external stirring force). This setup will prevent us from reaching any exact synchronization of the two fields because $\boldsymbol{u}_{\text {ref }}=\boldsymbol{u}$ is not a solution of Eq. (2) anymore, but it allows us to speak about a real-life problem. The absence of a forcing stirring term in Eq. (2) also implies that without nudging, the reconstructed flow would decay to zero monotonically, as we inject energy only by the information coming from the $\mathcal{I}\left(\boldsymbol{u}-\boldsymbol{u}_{\text {ref }}\right)$ term. Nudging, as is presented here, is thus a simple and unbiased assimilation-reconstruction scheme that is fully nonlinear and that can be understood as a generalized synchronization setup, as the dynamics that generated the data used [forced Navier-Stokes equations, i.e., Eq. (1)] is different from the one that drives the model [nudged Navier-Stokes, i.e., Eq. (2)]. For this reason, we focus on the problem of reconstructing small scales, not on reconstructing initial conditions. A proper assessment of the forecast quality would also require us to introduce an injection term. While this can be done, it is not within the scope of this paper. It is fair to point out though that a drawback of our setup is the inability to account for errors present in the model or data in the framework itself.

\section{B. Numerical protocols}

In our study, the reference true data $\boldsymbol{u}_{\text {ref }}$ is generated by numerically solving the Navier-Stokes equations (1), instead of using experimental measurements or field observations. The obvious advantage is that we can benchmark the reconstruction capabilities of nudging in a fully quantitative way, as we have access to the truth in every point in space and at every scale. Two different reference sets were produced, at medium and high Reynolds number [see Table I, where all the details of the numerical methods used to solve Eqs. (1) and (2) are given]. In the rest of the paper, all values are made dimensionless by fixing the kinetic energy, the size of the box, and the viscosity. The exact protocol adopted is the following. Starting from rest, we evolve Eq. (1) until the system reaches a stationary state (marking this moment as $t=0)$. Then we run for 10 turnover times (marking the final moment $t=T$ ), saving the fields at high frequency. We then solve Eq. (2) in the interval $t \in[0, T]$, using as initial condition $\mathcal{I} \boldsymbol{u}_{\text {ref }}(\boldsymbol{x}, t=0)$ and inputting the linearly interpolated field $\boldsymbol{u}_{\text {ref }}^{\tau}$ into the nudging term. This is done for different values of $\alpha$ and $\tau$, and for the different filters $\mathcal{I}$ (configuration Eulerian or Lagrangian or Fourier). The time step of both the reference and the nudged simulations is the same, and is chosen in accordance with the Courant-Friederichs-Lewy condition [2].

The implementation of the point-measurement-based filter, Eq. (3), is a bit delicate. As we do not have any other injection mechanism in Eq. (2), nudging only in points (i.e., one grid point) makes it difficult to inject
TABLE I. Parameters used for the different reference simulations experiments. All the respective nudged simulations have the same parameters. The code uses a two-step Adams-Bashfort scheme for the time integration, and the " $2 / 3$ rule" for dealiasing. The values listed are the total kinetic energy $E=1 / 2\left\langle\left|\boldsymbol{u}_{\text {ref }}\right|\right\rangle^{2}$, the Reynolds number $\operatorname{Re}=L(2 E)^{1 / 2} / \nu$, the viscosity $\nu$, the eddy turnover time $t_{L}=L /(2 E)^{1 / 2}$, the Kolmogorov timescale $t_{\eta}=\sqrt{\nu L /(2 E)^{3 / 2}}$, the Kolmogorov wave number $k_{\eta}=\left[\nu^{3} L /(2 E)^{3 / 2}\right]^{-1 / 4}$, and the number of grid points $N^{3}$. The largest scale of the flow $L$ is equal to $2 \pi$ in all simulations. In both cases $f_{\text {ref }}$ is a randomly generated, quenched in time, isotropic field with support on wave numbers with amplitudes $k \in[1,2]$ whose Fourier coefficients are given by $\hat{\boldsymbol{f}}_{\text {ref }}(\boldsymbol{k})=f_{0} k^{-7 / 2} e^{i \theta_{k}}$, where $\theta_{\boldsymbol{k}}$ are random in $[0,2 \pi)$ and $f_{0}=0.02$.

\begin{tabular}{lccccccc}
\hline \hline Type & $E_{\text {ref }}$ & $\operatorname{Re}$ & $\nu$ & $t_{L}$ & $t_{\eta}$ & $k_{\eta}$ & $N^{3}$ \\
\hline RUN1 & 1.20 & 3900 & 0.0025 & 4.06 & 0.065 & 78 & $256^{3}$ \\
RUN2 & 1.27 & 25000 & 0.0004 & 3.94 & 0.025 & 317 & $1024^{3}$ \\
\hline \hline
\end{tabular}

enough energy in order to maintain a stationary simulation with comparable Re. For this reason, we actually nudge in small spheres of radius $r=1.25 \eta$ centered around points $\boldsymbol{X}_{i}$. For the Eulerian setup, these points are always placed on a uniform equispaced three-dimensional grid covering the whole simulation box, so the only controlling parameter is the total number of probes $N_{p}$. The number we use to characterize each grid is the volume fraction:

$$
\phi=N_{p} \frac{(4 / 3) \pi r^{3}}{L^{3}},
$$

which is the ratio between the nudged and the total volumes. There are two useful wave numbers that can be defined:

$$
k_{l}=\frac{2 \pi N_{p}^{1 / 3}}{L}, \quad k_{r}=\frac{2 \pi}{r},
$$

where $k_{l}$ is associated with the minimum distance between probes and $k_{r}$ with the probe size. For the Lagrangian setup, the protocol is similar, with the only difference that the probe positions will move in time following the equation of a fluid tracer:

$$
\dot{\boldsymbol{X}}_{\boldsymbol{i}}(t)=\boldsymbol{u}_{\mathrm{ref}}\left(\boldsymbol{X}_{i}(t), t\right)
$$

Another important note to make is that, as the flow is incompressible, in configuration space nudging the nudging term $\mathcal{I}\left(\boldsymbol{u}-\boldsymbol{u}_{\text {ref }}\right)$ must be projected onto the incompressible modes via a Helmholtz decomposition in order for the flow to remain incompressible.

In Fig. 2 we give a first qualitative anticipation of both protocols, showing a 3D rendering of the reference field, of the probe distributions (nudging stations), and of the reconstructed flow for both Eulerian and Lagrangian 

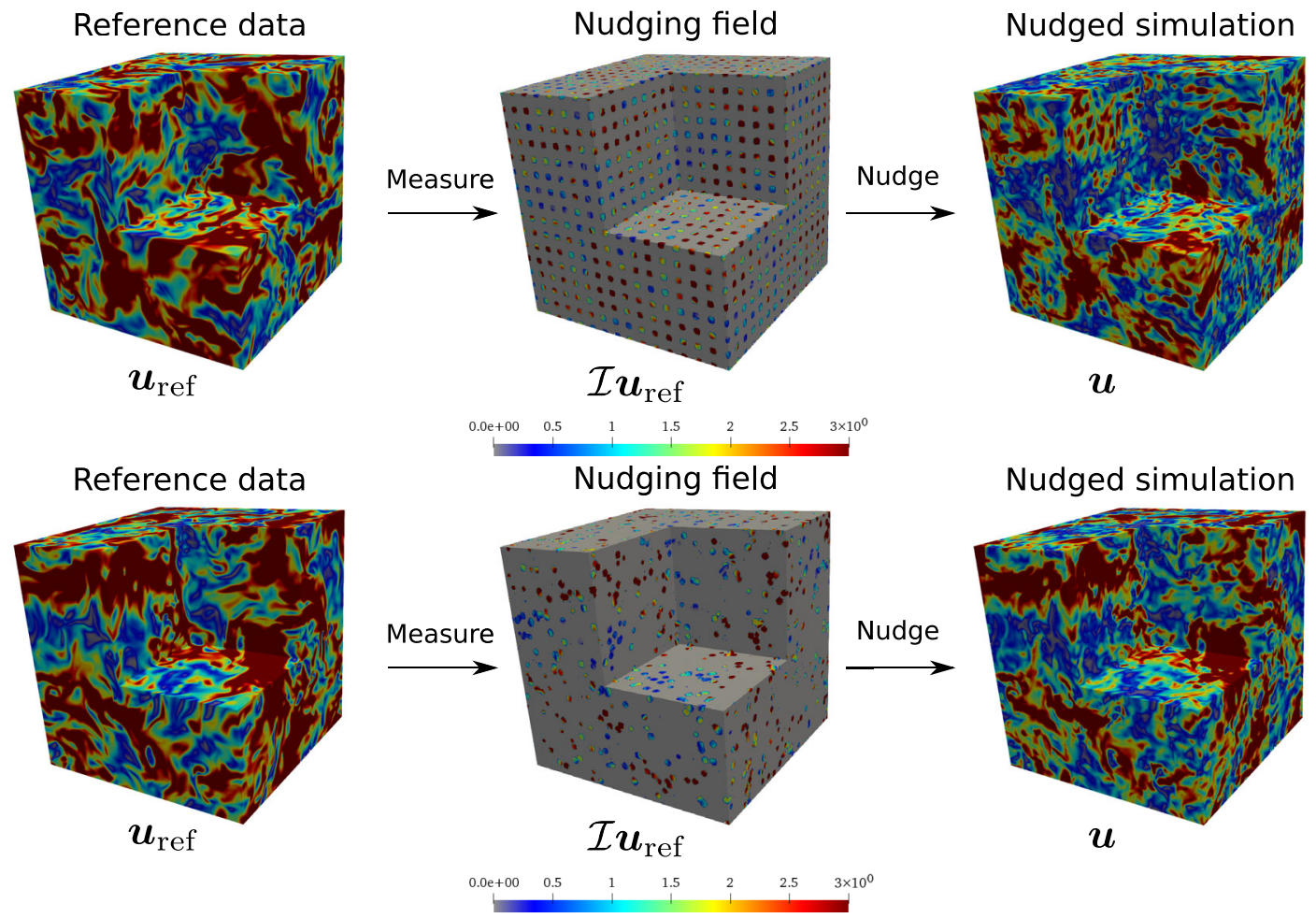

FIG. 2. Top: Visualizations of (left) reference fields $\boldsymbol{u}_{\text {ref, }}$, (middle) filtered or nudging fields $\mathcal{I} \boldsymbol{u}_{\text {ref }}$, and (right) nudged fields $\boldsymbol{u}$ for Eulerian nudging. The parameters are $\alpha t_{\eta}=6.5, \tau / t_{\eta}=1.5, \phi=0.05$. Bottom: Same as above but for Lagrangian nudging. The parameters are $\alpha t_{\eta}=6.5, \tau / t_{\eta}=1.5, \phi=0.03$. All flows are visualized at the final time of the simulations; see Sec. II B for more details.

nudging at high Reynolds number. As a third variation, we also explore nudging with spherical probes (placed on an Eulerian grid) where the velocity is fixed to have the value of a putative probe taken at the center of the computational volume, making the filtered field $\mathcal{I} \boldsymbol{u}_{\text {ref }}$ piecewise constant and mimicking the results from a localized reference field measurement. We refer to this scheme as "solid" nudging.

\section{Quantification of errors and correlations}

We start by defining the difference between the two fields (error field) at every space-time point:

$$
\boldsymbol{u}_{\Delta}(\boldsymbol{x}, t)=\boldsymbol{u}(\boldsymbol{x}, t)-\boldsymbol{u}_{\mathrm{ref}}(\boldsymbol{x}, t) .
$$

Then, in order to quantify the nudging performances for turbulent DA at both large and small scales, we define the relative errors in the point-to-point energy and enstrophy reconstruction, based on the time-averaged $L_{2}$ norm:

$$
\frac{E_{\Delta}}{E_{\text {ref }}}=\frac{\left\langle\left|\boldsymbol{u}_{\Delta}\right|^{2}\right\rangle}{\left\langle\left|\boldsymbol{u}_{\text {ref }}\right|^{2}\right\rangle}, \quad \frac{Z_{\Delta}}{Z_{\text {ref }}}=\frac{\left\langle\left|\boldsymbol{\omega}_{\Delta}\right|^{2}\right\rangle}{\left\langle\left|\boldsymbol{\omega}_{\text {ref }}\right|^{2}\right\rangle},
$$

where $\boldsymbol{\omega}=\boldsymbol{\nabla} \times \boldsymbol{u}$ is the vorticity field and the average is defined as the mean on the whole volume $V$ and on the whole experiment duration $T:\langle\bullet\rangle=1 /(T V) \int_{0}^{T} d t \int_{V} d \boldsymbol{x}(\bullet)$.
We sometimes look at the temporal variations, too, and in those cases we explicitly remark that what we are showing depends on time. So, for example, the time evolution of the energy of a nudge simulation will be referred to as $E(t)$.

In order to have a scale-by-scale control of the degree of synchronization, we introduce the energy spectrum of the difference between the nudged and the reference field, given by

$$
E_{\Delta}(k, t)=\frac{1}{2} \sum_{k \leq|k|<k+1}\left|\hat{\boldsymbol{u}}_{\Delta}(\boldsymbol{k}, t)\right|^{2} .
$$

The two most informative measures of the success of reconstruction at large (small) scales are based on velocity (vorticity) field correlations:

$$
\delta_{E}=\frac{\left\langle\boldsymbol{u} \cdot \boldsymbol{u}_{\mathrm{ref}}\right\rangle}{\langle|\boldsymbol{u}|\rangle\left\langle\left|\boldsymbol{u}_{\mathrm{ref}}\right|\right\rangle}, \quad \delta_{Z}=\frac{\left\langle\boldsymbol{\omega} \cdot \boldsymbol{\omega}_{\mathrm{ref}}\right\rangle}{\langle|\boldsymbol{\omega}|\rangle\left\langle\left|\boldsymbol{\omega}_{\mathrm{ref}}\right|\right\rangle} .
$$

Both quantities give account of how close the nudged and the reference fields are to each other, independently of their absolute values (see later), and do so by measuring the degree of instantaneous, not statistical, reconstruction. To clarify this, two realizations of the same flow that are widely separated in time would have the same statistical properties buy very low correlations (corresponding to $\delta=0)$. Evidently, we have 


$$
-1 \leq \delta_{E}, \delta_{Z} \leq 1
$$

As these quantities are time averages, we define the error associated with them as the standard deviation of the original time-varying signal.

Finally, it will be instructive to look also at the probability distribution function (PDF) of the pointwise error $\left|\boldsymbol{u}_{\Delta}(\boldsymbol{x}, t)\right|$ in order to understand specific issues connected to worstcase scenarios and/or whether there are spatial and/or topological structures that are better reconstructed. The latter point might not be so relevant for isotropic turbulence, but it is a key issue in nonisotropic conditions, like in the presence of boundaries or large-scale shear, as often happens in nature or in applied turbulent realizations.

\section{RESULTS}

\section{A. Nudging in configuration space}

We start by studying the case of nudging in configuration space, where the penalty term acts in confined regions in
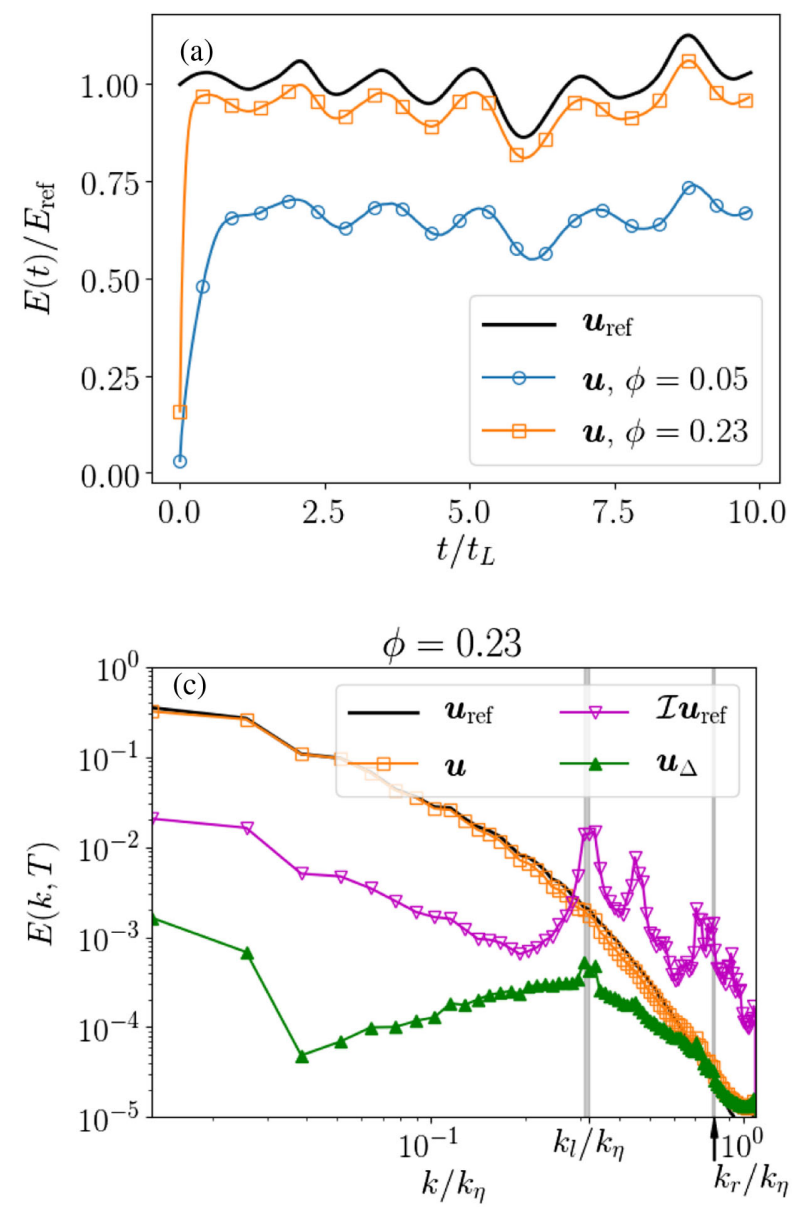

space. From Fig. 2 we see qualitatively that the nudged flows (right-hand panels) develop large-scale structures very close to the reference fields (left), even though nudging only acts locally. In this section we focus on the effects of varying the nudging amplitude $\alpha$ and the nudged volume fraction $\phi$. In all simulations, the temporal interpolation $\tau / t_{\eta}=1.5$ and only data from RUN1 are used (see Table I). We study the response to varying the timeinterpolation cadence $\tau$ in Sec. III B.

In Fig. 3(a) we show the evolution of the total energy for two nudged simulations, with $\phi=0.05$ and $\phi=0.23$. Both have $\alpha t_{\eta}=6.5$. The evolution of the total reference energy is also shown. As explained above, the initial condition for the nudged simulations is given by filtered reference at $t=0$, so they would look just like the middle panel in Fig. 2. It takes about one eddy turnover time for the nudged simulations to reach the stationary state, and to synchronize with the reference evolution, as seen in Fig. 3(a). The evolution of the energy shows some very

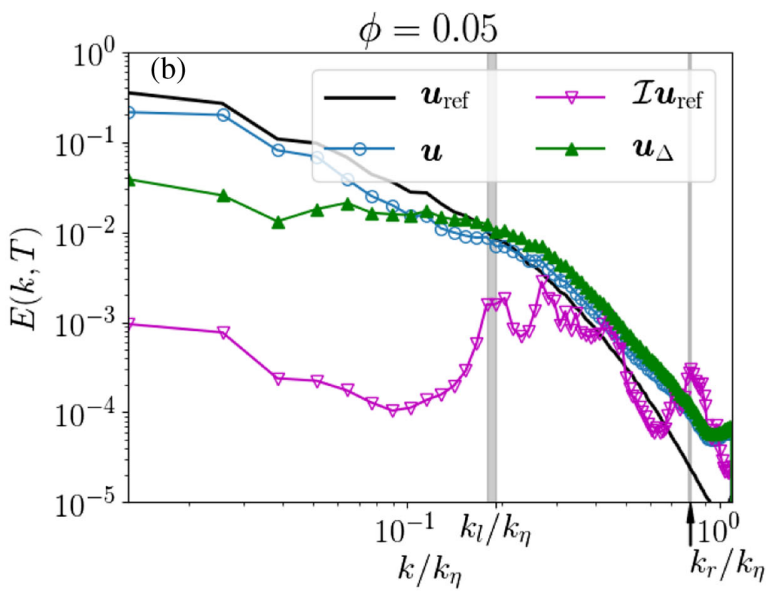

$\phi=0.05$

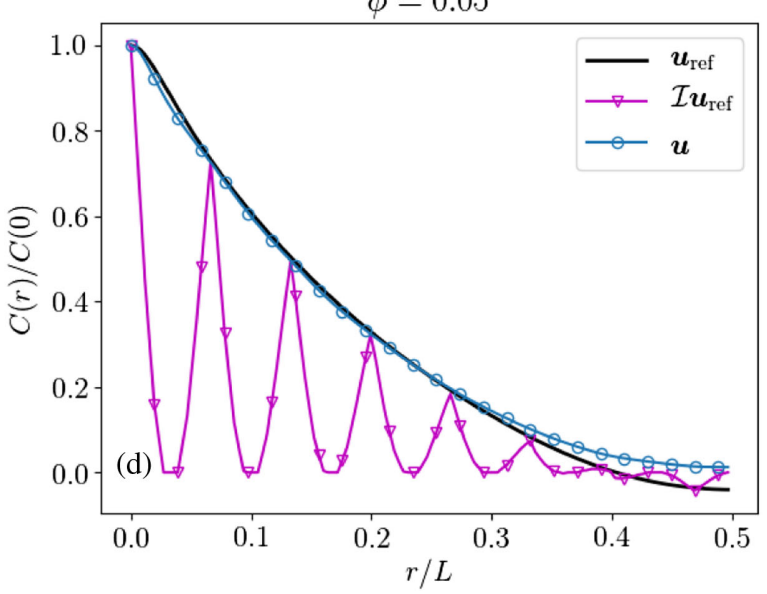

FIG. 3. (a) Evolution of the total energy for the reference field and for two nudged fields with different volume fraction, before and close to full synchronization, $\phi=0.05,0.23$. (b) Log-log plots of the energy spectra for the reference field (the truth) $\boldsymbol{u}_{\text {ref }}$ ), the nudging partial data $\mathcal{I} \boldsymbol{u}_{\text {ref }}$, the nudged field on the whole volume $\boldsymbol{u}$, and the spectrum of the error field $\boldsymbol{u}_{\Delta}$ at the end of the simulation for the simulation with $\phi=0.05$. (c) Same as (b) for the simulation with $\phi=0.23$. Gray regions mark the two typical wave numbers $k_{l}$ and $k_{r}$ (see text). Note the transition to full synchronization for (c), where the spectrum of the error field $\boldsymbol{u}_{\Delta}$ is negligible at all scales. (d) Twopoint correlation functions for the case with $\phi=0.05$. 

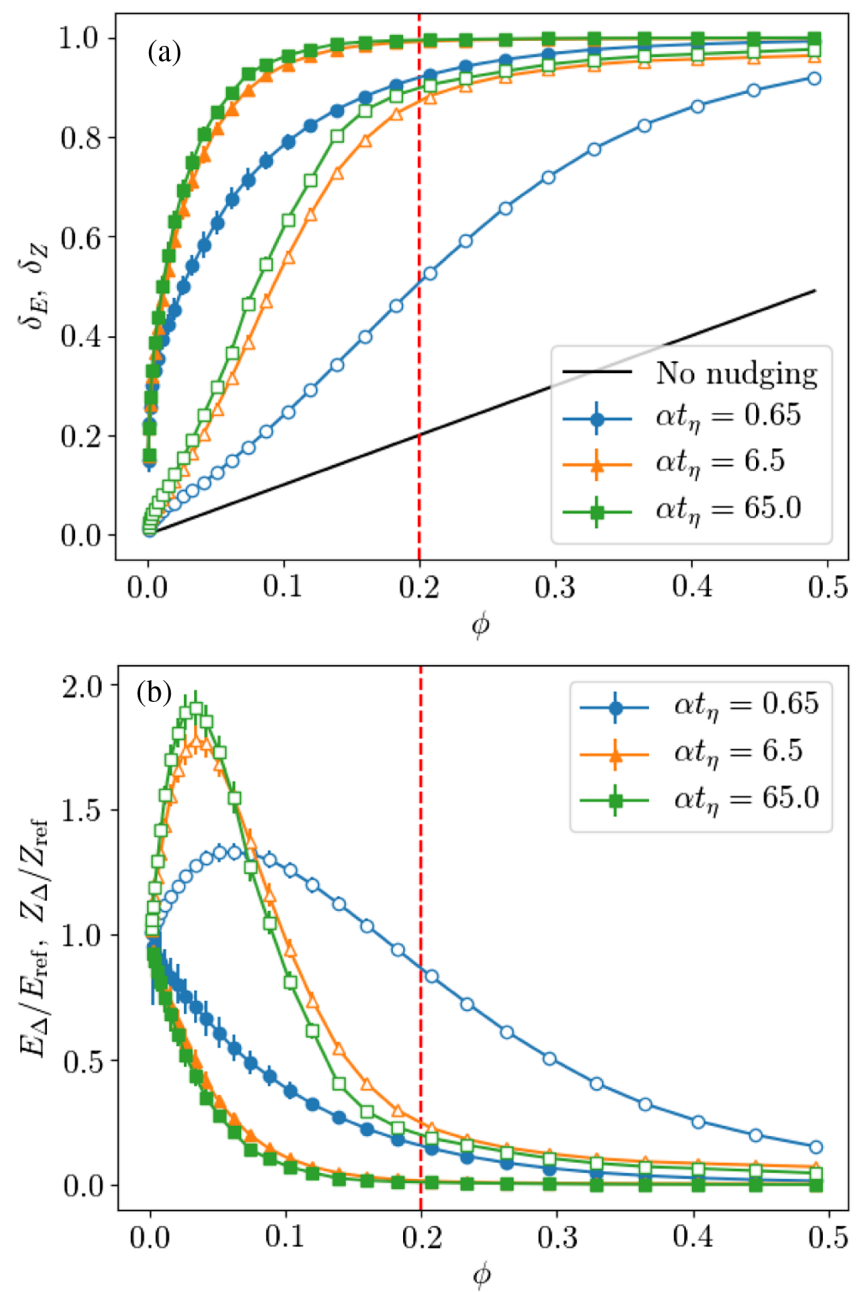

FIG. 4. (a) Values of the velocity correlations $\delta_{E}$ (filled symbols) and the vorticity correlations $\delta_{Z}$ (empty symbols). (b) Values of the energy error $E_{\Delta} / E_{\text {ref }}$ (filled symbols) and the enstrophy errors $Z_{\Delta} / Z_{\text {ref }}$ (empty symbols). All values are plotted as a function of the volume fraction $\phi$ and for different values of the nudging amplitude $\alpha$. Solid line in (a) represents the linear scaling expected for no effects of nudging (see text). The dashed red vertical lines mark the value $\phi_{c}=0.2$ as the typical estimate for transition to maximum synchronization in these setups. Here and in all figures error bars are always plotted; when they are not visible it means that they are smaller than the symbol size. See Sec. II C for the definitions of the different quantities and their errors.

interesting features. First, the energy of the nudged field is always smaller than that of the reference field. This is to be expected, as the only forcing term is the nudging term; thus the energy will be set by $\mathcal{I} \boldsymbol{u}_{\text {ref }}$ whose energy is always less than that of $\boldsymbol{u}_{\text {ref }}$. Second, nudging a higher volume fraction does indeed inject more energy and make the nudged system resemble the reference one more closely. It is important to remember that aside from the nudging term, no energy is being injected in the simulations as there is no external forcing mechanism present
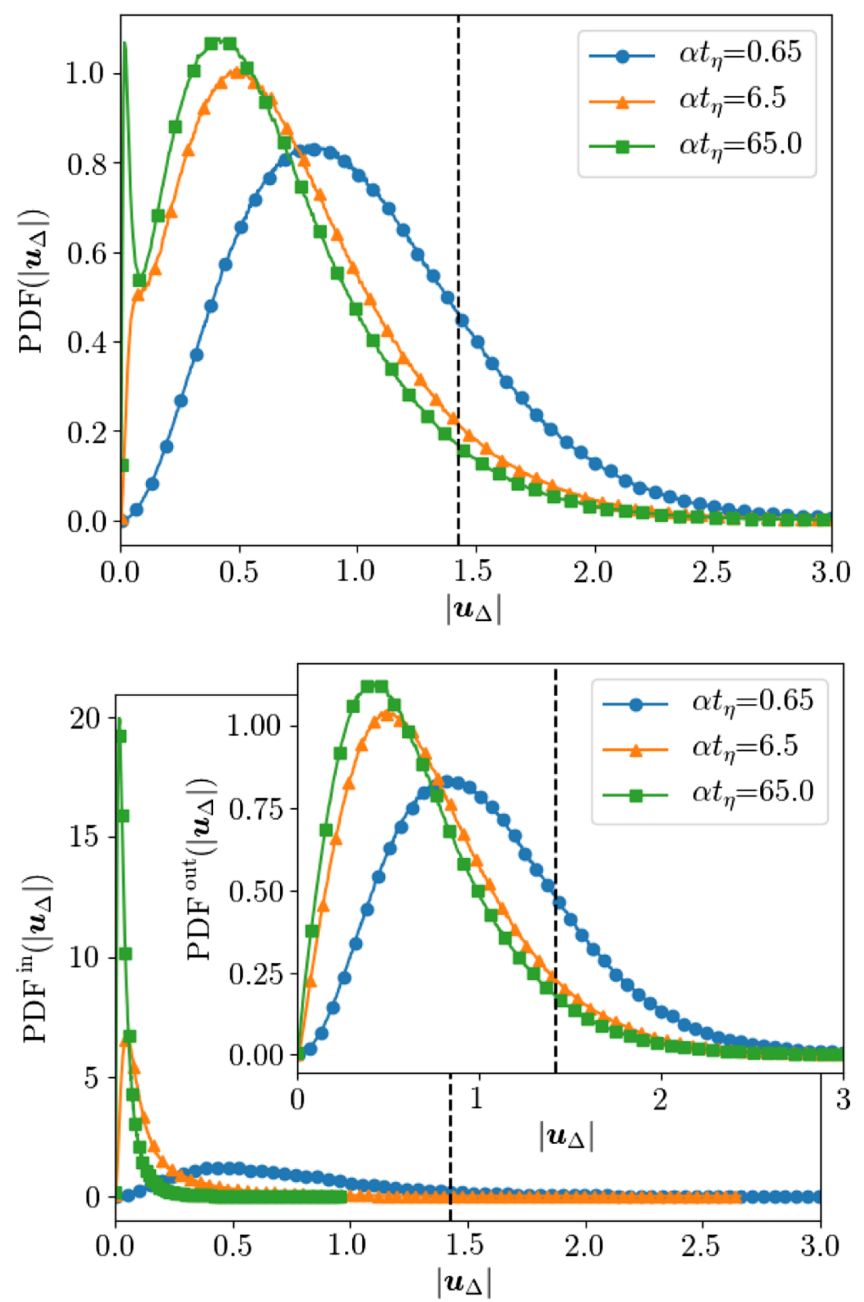

FIG. 5. Histograms of the point-by-point reconstructing error $\left|\boldsymbol{u}_{\Delta}\right|$ measured in the whole volume (top), only inside the nudging regions (bottom), and only outside (inset). The vertical dashed lines represent $\left\langle\left|\boldsymbol{u}_{\text {ref }}\right|\right\rangle$.

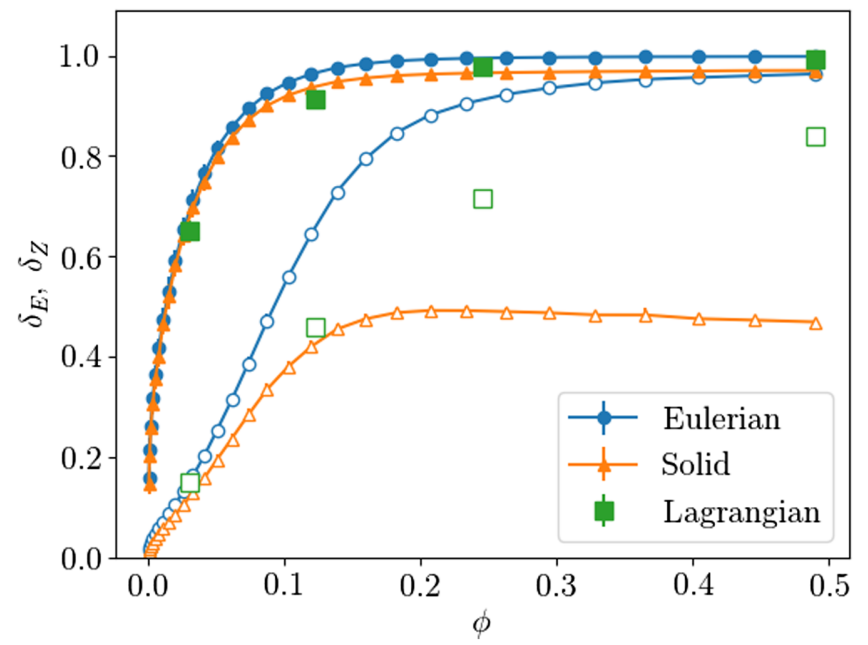

FIG. 6. Values of the velocity correlations $\delta_{E}$ (filled symbols) and the vorticity correlations $\delta_{Z}$ (empty symbols) as a function of the volume fraction $\phi$ for different nudging protocols. 

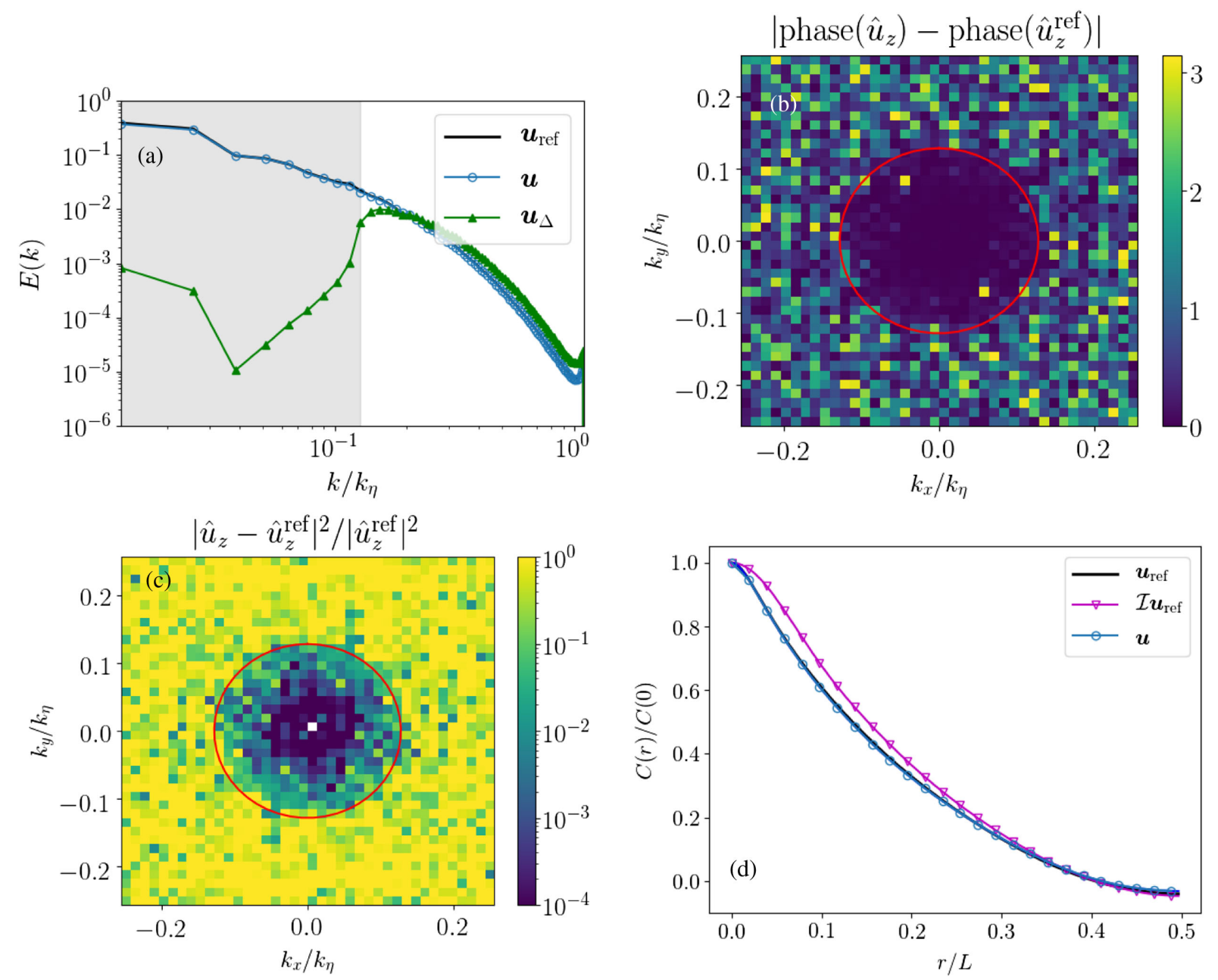

FIG. 7. (a) Energy spectra for the nudged $\boldsymbol{u}$, the reference $\boldsymbol{u}_{\text {ref }}$, and the error $\boldsymbol{u}_{\Delta}$ fields. The gray area indicates the nudged scales, $k<k_{n}$. (b) Point-by-point error between the phases of the nudged field and the reference one in the $k_{z}=0$ Fourier plane. (c) The same as for (b) but for the normalized amplitudes of the $z$ component. The red circle defines $k_{n}$. (d) Two-point correlation functions.

in Eq. (2). Third-and probably most striking-the nudged simulations are always able to follow the dynamical fluctuations of the reference field even in the presence of an appreciable amplitude mismatch. The latter is the indication that we can have good statistical correlations among the two fields without complete synchronization. This will be put into more quantitative terms below.

In Figs. 3(b) and 3(c) we compare the instantaneous energy spectra of (i) the total reference field $\boldsymbol{u}_{\text {ref }}$, (ii) the filtered reference field used for nudging $\mathcal{I} \boldsymbol{u}_{\text {ref }}$, (iii) the resulting nudged field $\boldsymbol{u}$ and the one which quantifies the synchronization error Eq. (11) for two different nudging volume fractions $\phi=0.05$ and $\phi=0.23$, respectively, all at time $T$, the final time of the simulations. First, let us note that the spectrum of $\mathcal{I} \boldsymbol{u}_{\text {ref }}$ is mainly concentrated at small scales (large wave numbers), with peaks located at the minimum distance between probes $k_{l}$ and at the probe size $k_{r}$, indicating that we are not supplying a large amount of information concerning the global large-scale motion (small wave numbers). In spite of the fact that most of the information provided is for the small scales, the scale-byscale synchronization error $E_{\Delta}(k, t)$ is smaller at large scales (small wave numbers) than at small scales (large wave numbers). A way to understand this is by looking at the two-point correlation function $C(r)=\left\langle u_{x}(\boldsymbol{x}) u_{x}(\boldsymbol{x}+r \hat{x})\right\rangle$, shown in Fig. 3(d) for the case with $\phi=0.05$. Evidently, some information on the correlations is input at almost every scale, and nudging is able to fill in the gaps. Furthermore, in the case with $\phi=0.23$ [Fig. 3(c)], the errors remain small across all scales, indicating a very good global reconstruction and a transition to full synchronization already for such relatively small volume fraction.

In Fig. 4 we show, for three different values of $\alpha$, the correlations $\delta_{E}$ and $\delta_{Z}$ given by Eq. (12) and the normalized errors $E_{\Delta} / E_{\text {ref }}, Z_{\Delta} / Z_{\text {ref }}$ given by Eq. (10) as a function of $\phi$. Good correlations and small relative errors in both the velocity and vorticity fields can be achieved with small nudged volume fractions. As one can see from Fig. 4(a), 


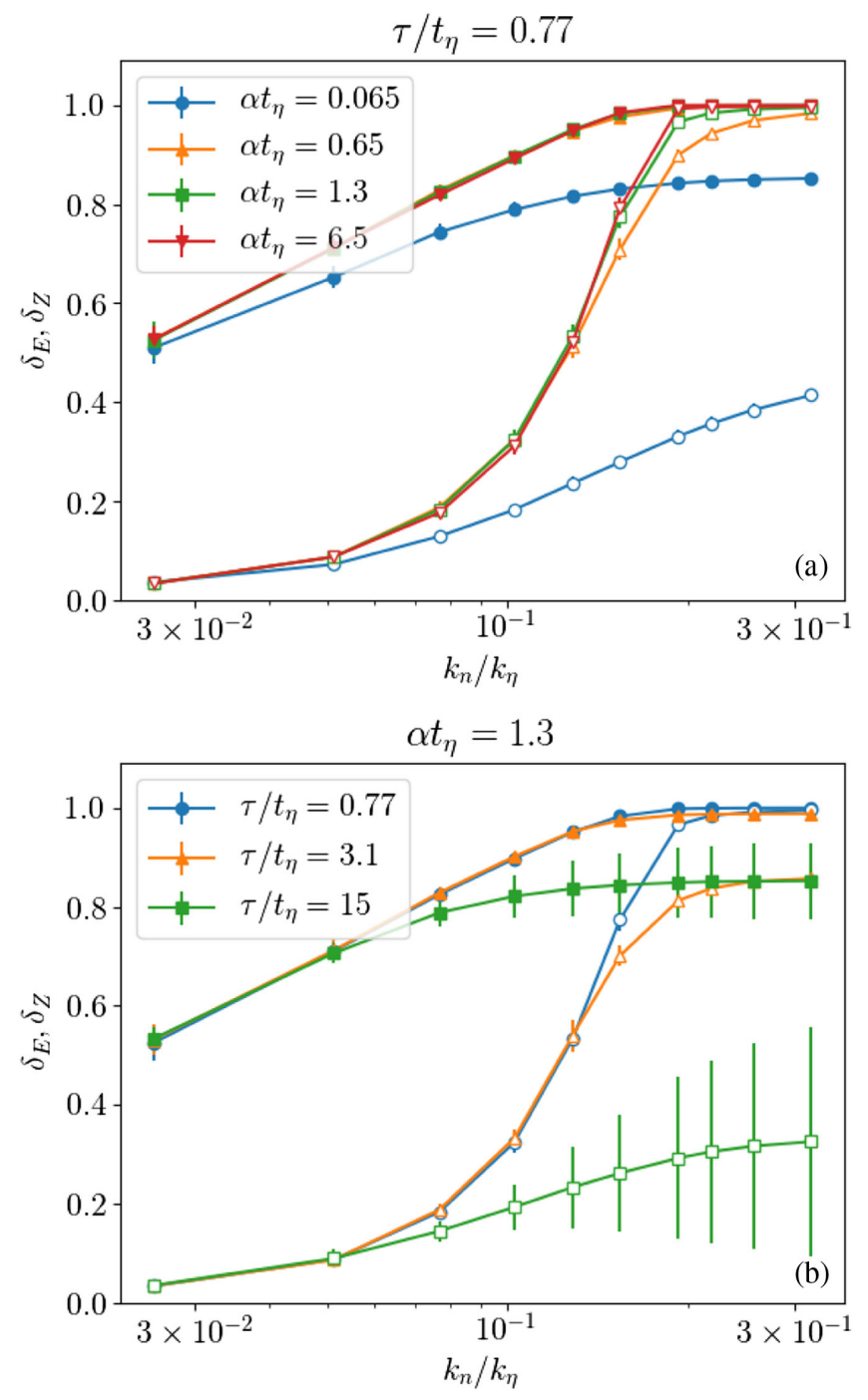

FIG. 8. Values of the velocity correlations $\delta_{E}$ (filled symbols) and the vorticity correlations $\delta_{Z}$ (empty symbols) as a function of the maximum nudged wave number $k_{n}$ for different values of the nudging amplitude $\alpha$ and the interpolation time $\tau$. In (a) $\tau / t_{\eta}=0.77$ and in (b) $\alpha t_{\eta}=1.3$.

already at $\phi \sim 0.2$ and for a nudging coefficient strong enough $\left(\alpha t_{\eta} \sim 5\right)$, we can reconstruct both total energy and total enstrophy with an accuracy close to $90 \%$. As expected, $\delta_{E}$ converges faster than $\delta_{Z}$, as it is determined by the large scales. For very small volume fractions, $\phi \ll 0.1$, the error is large, $E_{\Delta} / E_{\text {ref }}=Z_{\Delta} / Z_{\text {ref }}=1$, as the nudging field is almost equal to zero due to the fact that very little energy is injected into the system. As more energy is injected, the relative error in the enstrophy increases at the beginning while the one in the energy always decreases. This is because the velocity field can generate correlations more easily, while the vorticity field does not, so for $\phi \ll 0.1$, one gets

$$
\frac{Z_{\Delta}}{Z_{\text {ref }}}=\frac{\left\langle\left|\omega-\omega_{\text {ref }}\right|^{2}\right\rangle}{\left\langle\left|\omega_{\text {ref }}\right|^{2}\right\rangle} \approx \frac{\left\langle|\omega|^{2}\right\rangle+\mid\left\langle\left.\omega_{\text {ref }}\right|^{2}\right\rangle}{\left\langle\left|\omega_{\text {ref }}\right|^{2}\right\rangle} \approx 2,
$$

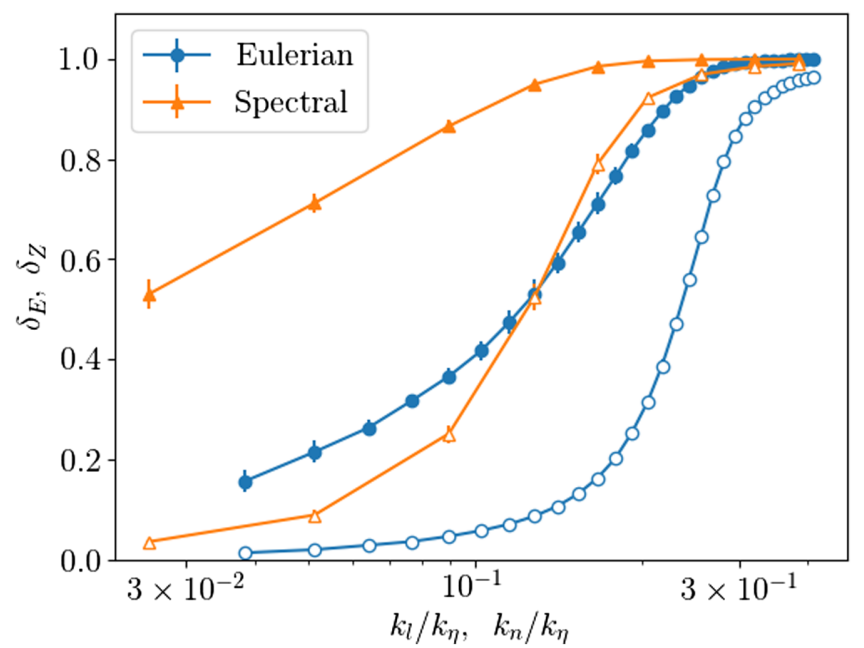

FIG. 9. Values of the velocity correlations $\delta_{E}$ (filled symbols) and the vorticity correlations $\delta_{Z}$ (empty symbols) as a function of the maximum nudged wave number $k_{n}$ (spectral case) or the mean nudged wavelength $k_{l}$ (Eulerian case).

where we have used that $\left\langle\boldsymbol{\omega} \cdot \boldsymbol{\omega}_{\text {ref }}\right\rangle \approx 0$ and that $\left\langle|\boldsymbol{\omega}|^{2}\right\rangle \approx$ $\left\langle\left|\omega_{\text {ref }}\right|^{2}\right\rangle$. By comparing the behavior for the three different values of $t_{\eta} \alpha$, one sees that by increasing $\alpha$ the transition to synchronization becomes sharper and little improvement is obtained as soon as $\alpha$ is of the same order of the highest frequency in the turbulent flow, $\sim 1 / t_{\eta}$. It should be noted that in the presence of noisy data, additional factors should be taken into account when choosing $\alpha$. Supposing that the nudging data are affected by some noise of typical amplitude $\epsilon$, this is equivalent to using a perfect data $\boldsymbol{u}_{\text {ref }}$ plus a forcing term of $\alpha \epsilon$ that acts only where the filter $\mathcal{I}$ is active [this can be seen by exchanging $\boldsymbol{u}_{\text {ref }}$ for $\boldsymbol{u}_{\text {ref }}+\epsilon$ in Eq. (2)]. A reasonable constraint would then be to have $\alpha \epsilon<\sqrt{2 E_{\text {ref }}} / t_{\eta}$.

All in all, the key parameter that drives the transition to synchronization is the volume fraction. We can estimate the critical value at which the level of reconstruction obtained saturates at the maximum level as

$$
\phi_{c} \sim 0.2 .
$$

In Fig. 4 we also plot the naive expectation obtained by supposing that nudging works only where we supply the information and gives fully uncorrelated results otherwise. In this case, the correlation coefficients would just scale as the volume fraction $\phi$ [solid line in Fig. 4(a)].

In Fig. 5 we show the PDF for the point-by-point error, $\left|\boldsymbol{u}_{\Delta}\right|=\left|\boldsymbol{u}-\boldsymbol{u}_{\text {ref }}\right|$, with the statistics taken over the whole volume (top panel), only inside the nudged regions (bottom panel), and only outside the nudged regions (inset), for different values of $\alpha t_{\eta}$ and with $\phi=0.05$. In accordance with Fig. 2, the errors inside the nudged regions are usually quite small, especially if compared with the mean $\left\langle\left|\boldsymbol{u}_{\text {ref }}\right|\right\rangle$, denoted by the dashed vertical line in each figure. The statistics outside the nudged regions dominate the statistics 

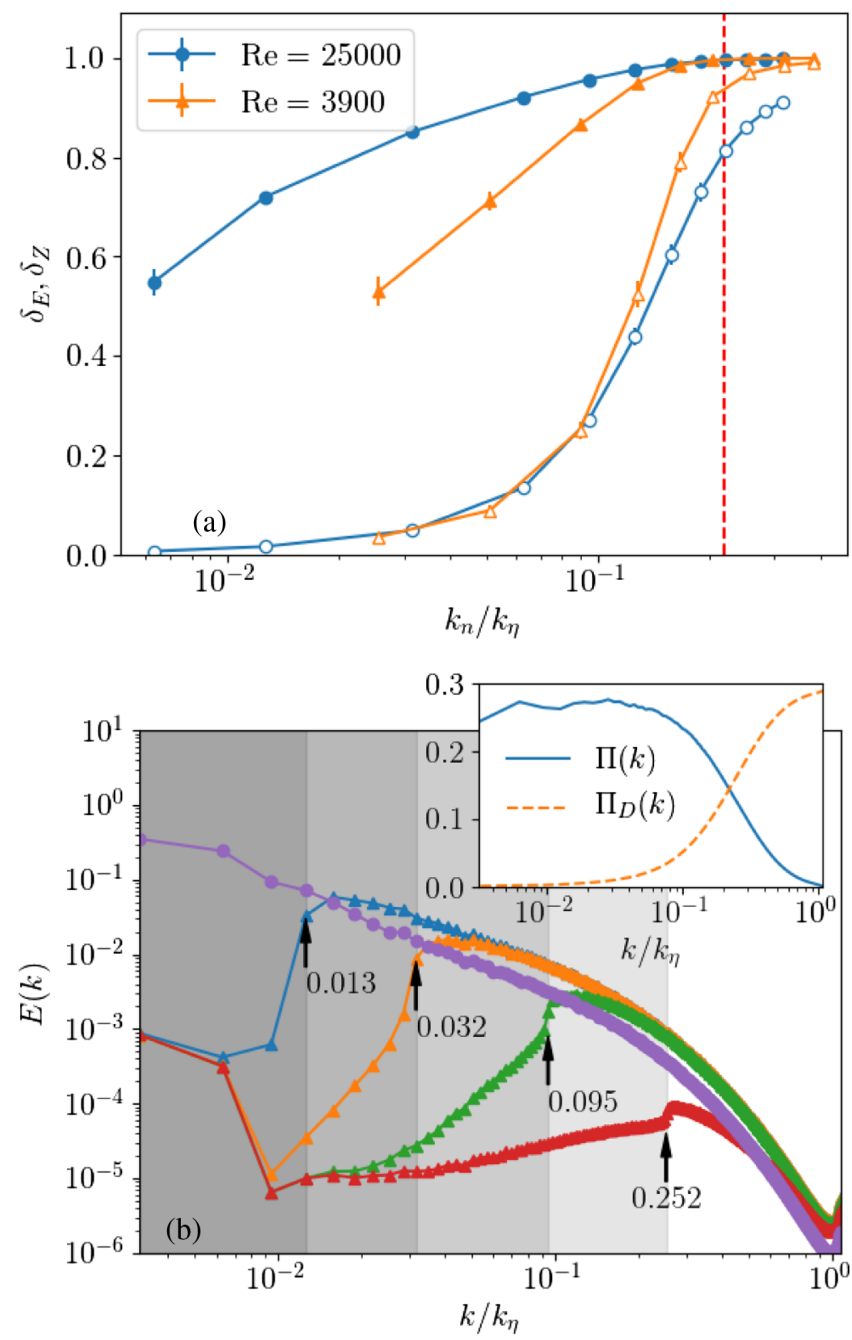

FIG. 10. (a) Values of the velocity correlations $\delta_{E}$ (filled symbols) and the vorticity correlations $\delta_{Z}$ (empty symbols) as a function of the maximum nudged wave number $k_{n}$ for different Reynolds numbers. Red dashed line marks the $k_{c} \sim 0.2 k_{\eta}$ value where transition to synchronization is obtained. (b) Energy spectra of the reference simulation RUN2 (round markers) and spectra of the difference field $u_{\Delta}$ at different $k_{n}$ (triangular markers). The value of $k_{n} / k_{\eta}$ for each nudged simulation is annotated with arrows. Inset: Nonlinear and viscous contributions to the energy fluxes for the reference simulation RUN2.

over the whole volume, as the volume fraction is small. Increasing $\alpha$ pushes the mean and the mode of the errors closer to zero, without producing any fat tails in the distribution. An analysis of the PDF of each field (not of the differences) can be found in our previous work [27].

Finally, in Fig. 6 we compare the three different ways of performing nudging in configuration space described in Sec. II A, Eulerian nudging, solid nudging, and Lagrangian nudging, by looking at $\delta_{E}$ and $\delta_{Z}$ as function of the nudging volume fraction. In all three cases, $\alpha t_{\eta}=6.5$ and $\tau / t_{\eta}=1.3$. As one can see from $\delta_{E}$, the velocity field gets well reconstructed by all schemes. On the other hand, $\delta_{Z}$ indicates that vorticity reconstruction does not work well for the "solid" schemes, as one could have expected because of the lack of small-scale information for this case. Surprisingly, also Lagrangian nudging performs slightly worse. One possible explanation is that the movement of the probes does not leave enough time for the flow synchronization at each point. One possible way to fix this problem could be to implement delayed-coordinates nudging, where the past history of the data is also used at each instant to guide the reconstruction, as was proposed for much simpler dynamical systems in Ref. [31], but has not been applied to turbulence until now.

\section{B. Nudging in Fourier space}

We now turn to characterizing how nudging in Fourier space works. We analyze the effects of varying the nudging amplitude $\alpha$, the interpolation time $\tau$, and the maximum nudged wave number $k_{n}$ in Eq. (5). To get a first glimpse of spectral nudging, we show in Fig. 7(a) the instantaneous energy spectrum for the full reference field $\boldsymbol{u}_{\text {ref }}$, that of the corresponding nudged field $\boldsymbol{u}$, and the scale-by-scale synchronization error $E_{\Delta}(k, t)$, for a simulation with $\alpha t_{\eta}=0.65, \tau / t_{\eta}=0.77$, and $k_{n} / k_{\eta}=0.13$. The gray region indicates the nudged window $k \in\left[0: k_{n}\right]$. Nudging is able to synchronize the nudged scales correctly, as seen by the fact that $E_{\Delta}(k, t)$ is very small for $k<k_{n}$, and also in Figs. 7(b) and 7(c), where the synchronization error for an instantaneous realization of Fourier phases and amplitudes is shown, respectively. The red circle in Figs. 7(b) and 7(c) denotes the maximum nudged wave number $k_{n}$. Finally, in Fig. 7(d) we show the two-point correlation functions $C(r)$ for this case. Concerning the transition to synchronization, we study now what happens when changing $k_{n}$. Figure 8 shows the equivalent of Fig. 4 but for Fourier nudging, i.e., $\delta_{E}$ and $\delta_{Z}$, as a function of $k_{n} / k_{\eta}$ for different values of $\alpha$ while keeping $\tau$ fixed [Fig. 8(a)], and for different values of $\tau$ while keeping $\alpha$ fixed Fig. 8(b)]. Velocity field correlations start at high values, already for small $k_{n}$, as the smallest wave numbers contain most of the energy, but vorticity field correlations require a larger number of modes to be nudged in order to build up. At around

$$
k_{n}=k_{c} \approx 0.2 k_{\eta},
$$

both $\delta_{E}$ and $\delta_{Z}$ show perfect synchronization being both equal to one.

By looking at Fig. 7(a), one recognizes that $k / k_{\eta}=0.2$ is around the end of the inertial range, indicating that one has to nudge everything but the viscous modes in order to reach the transition-to-synchronization limit. A similar result was found in Ref. [43], where, in contrast to our method, synchronization was studied by imposing the nudged modes to be equal to the reference ones (something similar to $\alpha \rightarrow \infty$ ) and by supplying also the exact external 

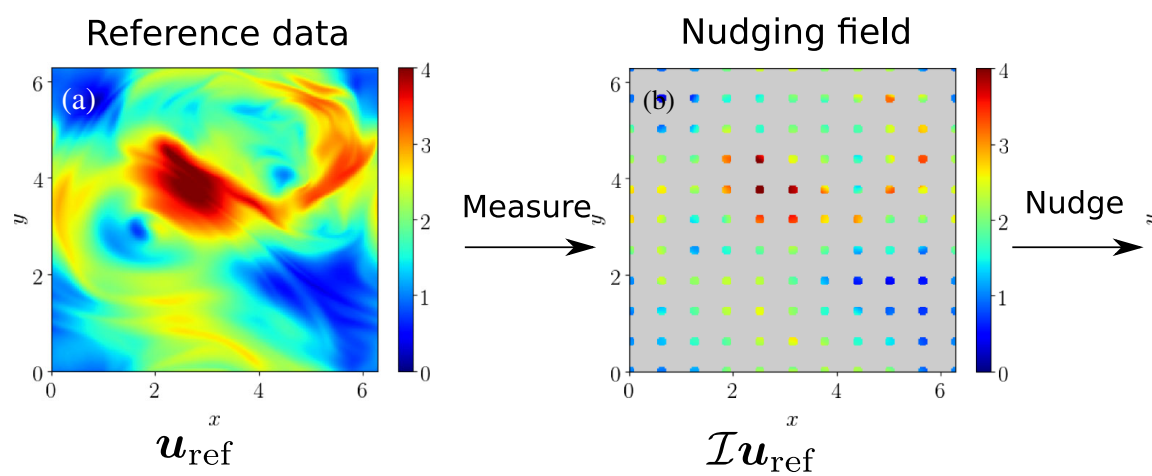

Nudged simulation

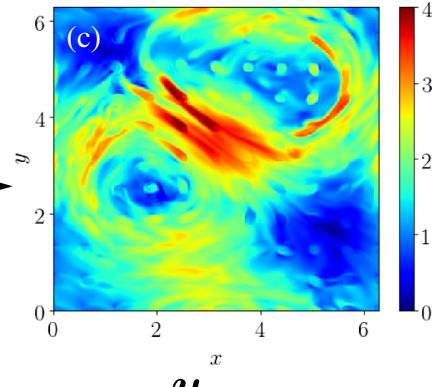

$\boldsymbol{u}$

FIG. 11. Visualizations of the local energy field of (a) a reference simulation $\boldsymbol{u}_{\text {ref }}$, (b) a filtered field $\mathcal{I} \boldsymbol{u}_{\text {ref }}$, (c) and a nudged simulation $\boldsymbol{u}$ of a rotating turbulent flow for $\phi=0.15, \alpha t_{\eta}=5.0$, and $\tau / t_{\eta}=2.0$. The reference simulation has $E_{\text {ref }}=1.3, \nu=0.002, N^{3}=256^{3}$, $L=2 \pi, t_{L}=3.89, t_{\eta}=0.05, \operatorname{Re}=5000$, and $\mathrm{Ro}=0.06$. The forcing $f_{\text {ref }}$ is a randomly generated, quenched in time, isotropic field with support on wave numbers with amplitudes $k \in[1,2]$ whose Fourier coefficients are given by $\hat{\boldsymbol{f}}_{\text {ref }}(\boldsymbol{k})=f_{0} k^{-7 / 2} e^{i \theta_{k}}$, where $\theta_{\boldsymbol{k}}$ are random in $[0,2 \pi)$ and $f_{0}=0.005$.

forcing field. It is important to remark that the number of d.o.f. necessary to control for full synchronization, $k_{c} \sim 0.2 k_{\eta}$, implies that the number of modes being nudged is still much smaller compared to the total number of d.o.f., around $1 \%$ actually, as the system is three dimensional. From Fig. 8 we also see that similar to the case of nudging in configuration space, increasing $\alpha$ has a positive effect (where the comment on the possible new constraints using noisy data would introduce mentioned above still holds). As expected, increasing $\tau$ negatively impacts the level of reconstruction, with lower values for the correlations and larger errors. The smaller the scales that we nudge, the more sensitive they become to the choice of $\tau$. This is because each Fourier mode has a characteristic correlation time, given the sweeping time $\tau_{s}(k) \sim 1 /(\sqrt{2 E} k)$ [64-66], that becomes shorter the higher the wave number. So if the correlation time of a particular mode becomes shorter than the interpolation time $\tau$, the interpolation starts to introduce unwanted errors.

In Fig. 9 we show the value of $\delta_{E}$ and $\delta_{Z}$ for Fourier nudging as a function of $k_{n} / k_{\eta}$ and for configuration space nudging as a function of $k_{l} / k_{\eta}$. The functional behavior is very similar, with the Fourier case expectedly doing much better than the Eulerian one for the large-scale reconstruction at small $k_{c}$ as information over the whole domain is used and not just in selected points. All this indicates that both Fourier and configuration d.o.f. play a similar role in driving the chaotic evolution of isotropic turbulence. In other words, there are no preferred leading variables that drive the global and local flow configuration. The situation can be obviously very different whenever the flow is driven by boundary effects, as in channel turbulence, external fields, as for convection and MHD, or influenced by the global setup, as for rotation (see Sec. III C). The fact that $k_{l}$ does serve as a good proxy for the effective scale at which Eulrian nudging is acting can also be used to choose an appropriate value for $\tau$, following the argument presented in the previous paragraph.
The effect of increasing the Reynolds number is studied in Fig. 10(a), where we compare velocity and vorticity correlations for RUN1 and RUN2 (see Table I) as a function of $k_{n} / k_{\eta}$. The fact that these two scans collapse on top of each other when plotting against $k_{n} / k_{\eta}$ shows that $k_{\eta}$ is the determining scale here. This can be understood better when looking at the energy spectra and flux. Figure 10(b) shows the energy spectra when nudging at different $k_{n}$ for the high Reynolds case. We see that when correlations are high, the spectra of the differences stays small for non-nudged wave numbers. The inset of the figure shows the nonlinear $\Pi(k)$ and dissipative $\Pi_{D}(k)$ contributions to the energy flux [55] for the reference simulation (RUN2). The value of $k_{n} / k_{\eta}$ for which synchronization is achieved is the same value at which the dissipation flux and the energy flux become equivalent, but it is smaller than that at which dissipation completely dominates. This certifies that one has to nudge all the scales dominated by inertial effects in order to have a complete synchronization of the nudged flow with respect to the reference data. It is important to note that the Reynolds number of RUN2 is quite high, especially compared to the standard simulations done in other studies of data assimilation [14-16].

\section{Nudging under the presence of large-scale structures}

Finally, we show the results of nudging a system where large-scale structures are present. As we mentioned in Sec. III A, it is reasonable to expect that different systems can show different sensitivity to a given nudging scheme. Homogeneous and isotropic turbulence can be considered the worst-case scenario as it lacks large-scale coherent structures. In order to show that nudging can indeed be more efficient in the presence of some coherency in the system, we applied it to a rotating turbulent flow. Rotating turbulence is known for generating large columnar vortices 


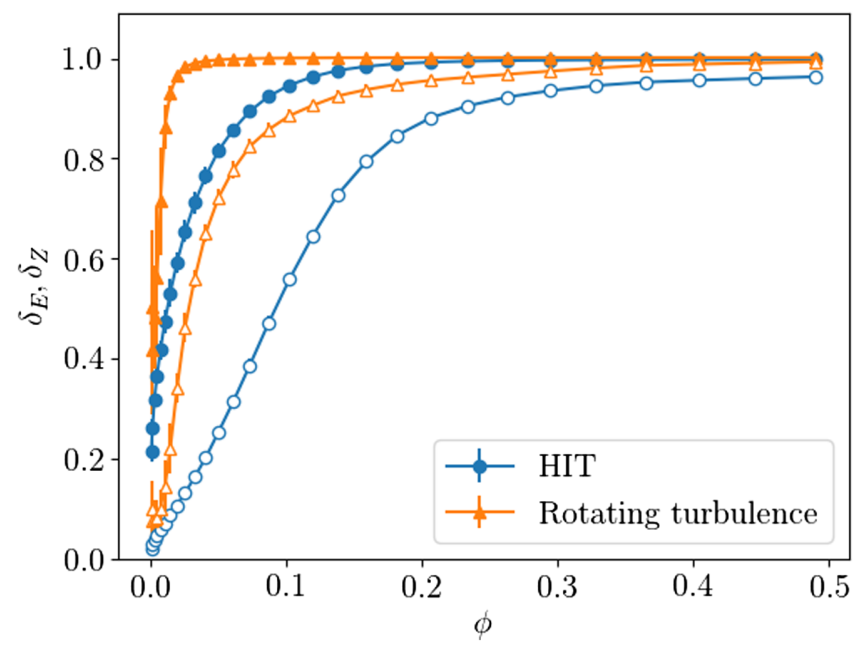

FIG. 12. Values of the velocity correlations $\delta_{E}$ and the vorticity correlations $\delta_{Z}$ (empty symbols) as a function of the volume fraction $\phi$ for homogeneous and isotropic turbulence (HIT) or under rotation. The HIT simulations have $\alpha t_{\eta}=6.5$ and $\tau / t_{\eta}=1.5$, and the rotating turbulence ones have $\alpha t_{\eta}=5$ and $\tau / t_{\eta}=2.0$.

with a strong translational symmetry in the direction parallel to the rotation axis [1,67-69]. It is known that nudging can reconstruct the inverse cascade present in rotating flows [27], although this was shown only for spectral nudging. Equations (1) and (2) were modified by adding to the right-hand side a Coriolis term of the form $-2 \Omega \hat{z} \times \boldsymbol{u}$, with $\Omega$ being the rotation frequency (see caption of Fig. 11 for more details on the simulations). Figure 11 shows a visualization of horizontal slices of the energy of the full reference field, of the filtered or nudging field, and of the nudged field with applications of the protocol on the configuration domain. The aforementioned large-scale structures are quite easy to spot, and it is evident how nudging works better in this scenario. Figure 12 compares the value of $\delta_{E}$ and $\delta_{Z}$ for one of the previous cases with nudging homogeneous isotropic turbulence and one case of nudging rotating turbulence. When the flow is under rotation, nudging is able to synchronize both the velocity and vorticity fields to the reference data at much lower volume fractions. This indicates that nudging can be a very powerful tool in problems that have large-scale structures but are still nonlinear and chaotic.

\section{CONCLUSIONS}

We have presented the first systematic application of nudging to three-dimensional homogeneous and isotropic turbulence for big-data assimilation (high Reynolds number regime). We have investigated the transition to full or scale-by-scale synchronization when changing the quantity and the quality (type) of information used. In particular, we have implemented nudging with measurements of (i) field values on a fixed number of spatial locations (Eulerian case), (ii) Fourier coefficients of the fields on a fixed range of wave numbers (Fourier case), or (iii) field values along a set of moving probes inside the flow (Lagrangian case). Concerning the quantity of information, we have shown that full synchronization is achieved as soon as the number of d.o.f. supplied by the nudging field covers a range of scales that is about one-quarter of the dissipative Kolmogorov wave number (i.e., the largest wave number where nonlinear inertial d.o.f. are still active), coinciding with the scale at which inertial and viscous fluxes match each other. We have tested this at both moderate and high Reynolds numbers, where $k_{\eta} \sim k_{0} \mathrm{Re}^{3 / 4}$, and $k_{0}$ is the energy containing scale. Similarly for nudging in configuration space, the critical volume fraction to reach synchronization is $\phi_{c} \sim 0.2$. We have found that nudging in Fourier space improves data reconstruction, although paying the price that it is more difficult to apply in realistic field-data applications. Concerning the quality of information, we have found that inputting Lagrangian data tends to deteriorate the ability to reconstruct but opens a much more flexible tool for environmental applications. Our Lagrangian approach differs from the usual Lagrangian data assimilation [13,70], as we are not nudging or assimilating a tracer's position, we are only using the information along its trajectory. This situation can be improved by implementing a delay-coordinate nudging scheme [31], where information on the time evolution of each particle over a specified time window is used. It is also important to note that the fields we reconstruct have many points (on the order of $10^{7}$ ), so at high volume fractions, applying a smooth three-dimensional interpolation scheme in order to try to reconstruct the fields could be prohibitively expensive. Finally, we applied nudging to a turbulent rotating flow and showed that, despite the dynamics being richer with a split forward and backward energy cascade [1,2,55], the presence of large-scale coherent structures helps nudging to reconstruct the reference flow at lower volume fractions than in the isotropic case, an important fact for many potential applications.

It is important to remark that our implementation of nudging is different from the usual one, because we do not supply information about the external forcing mechanisms in the nudged field evolution. This is done on purpose, to broaden its applicability to realistic conditions that are often encountered in the lab or in the open fields. Also for this reason, the focus of this work is on reconstructing small scales, not initial conditions, as any forward unnudged simulation would require some injection mechanism. Adding a forcing mechanism is, of course, possible and will be the subject of our future work. It is safe to say though that nudged solutions will necessarily be better than just using the supplied data, especially when full-scale synchronization occurs. Furthermore, the application of nudging to big data goes well beyond the 
data assimilation scope, as it can be seen as an unbiased equation-informed tool for classification of complex fields [27] and/or as a tool to highlight a hierarchy of correlations inside fluid turbulent applications, thanks to the mapping from input to output data mediated by the equations of motion. For example, it is tempting to imagine that nudging could be used in thermal Rayleigh-Bénard convection and in MHD to understand the casual correlation between temperature or magnetic field with the velocity field and in bounded flows to disentangle the relative importance of near-wall versus bulk regions for driving the scale and location dependent turbulent fluctuations. Work in this direction will be reported elsewhere. Finally, this study must be seen as a first step toward a more systematic sideby-side comparison of different data assimilation techniques to fully developed turbulent flows, and work in such direction will certainly be highly beneficial for the communities working in geosciences and fundamental and applied fluid dynamics.

\section{ACKNOWLEDGMENTS}

The authors acknowledge partial funding from the European Research Council under the European Community's Seventh Framework Program, ERC Grant Agreement No. 339032. The authors acknowledge help from Michele Buzzicotti with the Lagrangian simulations. We acknowledge Francesco Borra, Massimo Cencini, Nathan Glatt-Holtz, Cecilia Freire Mondaini, Angelo Vulpiani, Mengze Wang, and Tamer Zaki for useful discussions.

[1] P. A. Davidson, Turbulence in Rotating, Stratified and Electrically Conducting Fluids (Cambridge University Press, Cambridge, England, 2013).

[2] S. B. Pope, Turbulent Flows (Cambridge University Press, Cambridge, England, 2000).

[3] E. Kalnay, Atmospheric Modeling, Data Assimilation and Predictability (Cambridge University Press, Cambridge, England, 2003).

[4] R. N. Bannister, A Review of Operational Methods of Variational and Ensemble-Variational Data Assimilation, Q. J. R. Meteorol. Soc. 143, 607 (2017).

[5] A. Carrassi, M. Bocquet, L. Bertino, and G. Evensen, Data Assimilation in the Geosciences: An Overview of Methods, Issues, and Perspectives, Wiley Interdiscip. Rev. Clim. Change 9, e535 (2018).

[6] P. Bauer, A. Thorpe, and G. Brunet, The Quiet Revolution of Numerical Weather Prediction, Nature (London) 525, 47 (2015).

[7] O. Talagrand and P. Courtier, Variational Assimilation of Meteorological Observations with the Adjoint Vorticity Equation. I: Theory, Q. J. R. Meteorol. Soc. 113, 1311 (1987).
[8] S. K. Park and D. Županski, Four-Dimensional Variational Data Assimilation for Mesoscale and Storm-Scale Applications, Meteorol. Atmos. Phys. 82, 173 (2003).

[9] F. Rawlins, S. P. Ballard, K. J. Bovis, A. M. Clayton, D. Li, G. W. Inverarity, A. C. Lorenc, and T. J. Payne, The Met Office Global Four-Dimensional Variational Data Assimilation Scheme, Q. J. R. Meteorol. Soc. 133, 347 (2007).

[10] G. Evensen, Sequential Data Assimilation with a Nonlinear Quasi-Geostrophic Model Using Monte Carlo Methods to Forecast Error Statistics, J. Geophys. Res. Oceans 99, 10143 (1994).

[11] G. Evensen, Data Assimilation: The Ensemble Kalman Filter (Springer Science \& Business Media, Berlin, 2006).

[12] P. L. Houtekamer and F. Zhang, Review of the Ensemble Kalman Filter for Atmospheric Data Assimilation, Mon. Weather Rev. 144, 4489 (2016).

[13] M. Nodet, Variational Assimilation of Lagrangian Data in Oceanography, Inverse Probl. 22, 245 (2006).

[14] T. R. Bewley and B. Protas, Skin Friction and Pressure: The Footprints of Turbulence, Physica (Amsterdam) 196D, 28 (2004).

[15] T. Suzuki, Reduced-Order Kalman-Filtered Hybrid Simulation Combining Particle Tracking Velocimetry and Direct Numerical Simulation, J. Fluid Mech. 709, 249 (2012).

[16] T. Suzuki and Y. Hasegawa, Estimation of Turbulent Channel Flow at $\operatorname{Re}_{\tau}=100$ Based on the Wall Measurement Using a Simple Sequential Approach, J. Fluid Mech. 830, 760 (2017).

[17] V. Mons, Q. Wang, and T. A. Zaki, Kriging-Enhanced Ensemble Variational Data Assimilation for Scalar-Source Identification in Turbulent Environments, J. Comput. Phys. 398, 108856 (2019).

[18] D. Leutwyler, O. Fuhrer, X. Lapillonne, D. Lthi, and C. Schr, Towards European-Scale Convection-Resolving Climate Simulations with GPUs: A Study with COSMO 4.19, Geosci. Model Dev. 9, 3393 (2016).

[19] J.-I. Yano, M. Z. Ziemiaski, M. Cullen, P. Termonia, J. Onvlee, L. Bengtsson, A. Carrassi, R. Davy, A. Deluca, S. L. Gray, V. Homar, M. Khler, S. Krichak, S. Michaelides, V. T. J. Phillips, P. M. M. Soares, and A. A. Wyszogrodzki, Scientific Challenges of Convective-Scale Numerical Weather Prediction, Bull. Am. Meteorol. Soc. 99, 699 (2018).

[20] N. J. Gordon, D. J. Salmond, and A. F. M. Smith, Novel Approach to Nonlinear/Non-Gaussian Bayesian State Estimation, IEE Proc. Radar Signal Process. 140, 107 (1993).

[21] P. J. van Leeuwen and M. Ades, Efficient Fully Nonlinear Data Assimilation for Geophysical Fluid Dynamics, Comput. Geosci. 55, 16 (2013).

[22] J. Poterjoy, R. A. Sobash, and J. L. Anderson, ConvectiveScale Data Assimilation for the Weather Research and Forecasting Model Using the Local Particle Filter, Mon. Weather Rev. 145, 1897 (2017).

[23] K. Fukami, K. Fukagata, and K. Taira, Super-resolution Reconstruction of Turbulent Flows with Machine Learning, J. Fluid Mech. 870, 106 (2019).

[24] J. L. Callaham, K. Maeda, and S. L. Brunton, Robust Flow Reconstruction from Limited Measurements via Sparse Representation, Phys. Rev. Fluids 4, 103907 (2019). 
[25] J. E. Hoke and R. A. Anthes, The Initialization of Numerical Models by a Dynamic-Initialization Technique, Mon. Weather Rev. 104, 1551 (1976).

[26] S. Lakshmivarahan and J. M. Lewis, Nudging Methods: A Critical Overview, in Data Assimilation for Atmospheric, Oceanic and Hydrologic Applications (Springer, Berlin, 2013), Vol. II, pp. 27-57.

[27] P. C. Di Leoni, A. Mazzino, and L. Biferale, Inferring Flow Parameters and Turbulent Configuration with PhysicsInformed Data Assimilation and Spectral Nudging, Phys. Rev. Fluids 3, 104604 (2018).

[28] U. Frisch, Turbulence: The Legacy of A.N. Kolmogorov (Cambridge University Press, Cambridge, England, 1995).

[29] D. Auroux and J. Blum, A Nudging-Based Data Assimilation Method: The Back and Forth Nudging (BFN) Algorithm, Nonlinear Processes Geophys. 15, 305 (2008).

[30] H. Du and L. A. Smith, Pseudo-orbit Data Assimilation. Part I: The Perfect Model Scenario, J. Atmos. Sci. 71, 469 (2014).

[31] D. Pazó, A. Carrassi, and J. M. Lpez, Data Assimilation by Delay-Coordinate Nudging, Q. J. R. Meteorol. Soc. 142, 1290 (2016).

[32] H. von Storch, H. Langenberg, and F. Feser, A Spectral Nudging Technique for Dynamical Downscaling Purposes, Mon. Weather Rev. 128, 3664 (2000).

[33] K. M. Waldron, J. Paegle, and J. D. Horel, Sensitivity of a Spectrally Filtered and Nudged Limited-Area Model to Outer Model Options, Mon. Weather Rev. 124, 529 (1996).

[34] G. Miguez-Macho, G. L. Stenchikov, and A. Robock, Spectral Nudging to Eliminate the Effects of Domain Position and Geometry in Regional Climate Model Simulations, J. Geophys. Res. Atmos. 109, D13104 (2004).

[35] A. Farhat, E. Lunasin, and E. S. Titi, Abridged Continuous Data Assimilation for the 2D Navier-Stokes Equations Utilizing Measurements of Only One Component of the Velocity Field, J. Math. Fluid Mech. 18, 1 (2016).

[36] M. Gesho, E. Olson, and E. S. Titi, A Computational Study of a Data Assimilation Algorithm for the Two-Dimensional Navier-Stokes Equations, Commun. Comput. Phys. 19, 1094 (2016).

[37] C. Foias, C. Mondaini, and E. Titi, A Discrete Data Assimilation Scheme for the Solutions of the Two-Dimensional Navier-Stokes Equations and Their Statistics, SIAM J. Appl. Dyn. Syst. 15, 2109 (2016).

[38] A. Biswas, C. Foias, C. F. Mondaini, and E. S. Titi, Downscaling Data Assimilation Algorithm with Applications to Statistical Solutions of the Navier-Stokes Equations, arXiv: 1711.04067.

[39] D. A. F. Albanez, H. J. Nussenzveig Lopes, and E. S. Titi, Continuous Data Assimilation for the Three-Dimensional Navier-Stokes- $\alpha$ Model, Asymptotic Analysis 97, 139 (2016).

[40] A. Farhat, H. Johnston, M. S. Jolly, and E. S. Titi, Assimilation of Nearly Turbulent Rayleigh-Bénard Flow through Vorticity or Local Circulation Measurements: A Computational Study, arXiv:1709.02417.

[41] A. Farhat, N. E. Glatt-Holtz, V. R. Martinez, S. A. McQuarrie, and J.P. Whitehead, Data Assimilation in Large-Prandtl Rayleigh-Bénard Convection from Thermal Measurements, arXiv:1903.01508.
[42] A. Carrassi, M. Ghil, A. Trevisan, and F. Uboldi, Data Assimilation as a Nonlinear Dynamical Systems Problem: Stability and Convergence of the Prediction-Assimilation System, Chaos 18, 023112 (2008).

[43] C. C. Lalescu, C. Meneveau, and G. L. Eyink, Synchronization of Chaos in Fully Developed Turbulence, Phys. Rev. Lett. 110, 084102 (2013).

[44] S. L. Brunton, J. L. Proctor, and J. N. Kutz, Discovering Governing Equations from Data by Sparse Identification of Nonlinear Dynamical Systems, Proc. Natl. Acad. Sci. U.S.A. 113, 3932 (2016).

[45] S. H. Rudy, S. L. Brunton, J. L. Proctor, and J. N. Kutz, Data-Driven Discovery of Partial Differential Equations, Sci. Adv. 3, e1602614 (2017).

[46] M. Raissi, P. Perdikaris, and G. E. Karniadakis, Physics Informed Deep Learning (Part I): Data-Driven Solutions of Nonlinear Partial Differential Equations, arXiv:1711 .10561.

[47] M. Raissi, P. Perdikaris, and G. E. Karniadakis, Physics Informed Deep Learning (Part II): Data-Driven Discovery of Nonlinear Partial Differential Equations, arXiv:1711. 10566.

[48] I. Cialenco and N. Glatt-Holtz, Parameter Estimation for the Stochastically Perturbed Navier-Stokes Equations, Stoch. Proc. Appl. 121, 701 (2011).

[49] H. Du and L. A. Smith, Parameter Estimation through Ignorance, Phys. Rev. E 86, 016213 (2012).

[50] M. Bocquet, J. Brajard, A. Carrassi, and L. Bertino, Data Assimilation as a Learning Tool to Infer Ordinary Differential Equation Representations of Dynamical Models, Nonlinear Processes Geophys. 26, 143 (2019).

[51] J. Pathak, B. Hunt, M. Girvan, Z. Lu, and E. Ott, ModelFree Prediction of Large Spatiotemporally Chaotic Systems from Data: A Reservoir Computing Approach, Phys. Rev. Lett. 120, 024102 (2018).

[52] K. Nakai and Y. Saiki, Machine-Learning Prediction of Fluid Variables from Data Using Reservoir Computing, Phys. Rev. E 98, 023111 (2018).

[53] A. Mohan, D. Daniel, M. Chertkov, and D. Livescu, Compressed Convolutional LSTM: An Efficient Deep Learning Framework to Model High Fidelity 3D Turbulence, arXiv:1903.00033.

[54] J. Brajard, A. Carrassi, M. Bocquet, and L. Bertino, Combining Data Assimilation and Machine Learning to Emulate a Dynamical Model from Sparse and Noisy Observations: A Case Study with the Lorenz 96 Model, https://doi.org/10.5194/gmd-2019-136.

[55] A. Alexakis and L. Biferale, Cascades and Transitions in Turbulent Flows, Phys. Rep. 767-769, 1 (2018).

[56] J. Kim, P. Moin, and R. Moser, Turbulence Statistics in Fully Developed Channel Flow at Low Reynolds Number, J. Fluid Mech. 177, 133 (1987).

[57] J. Hwang, J. Lee, H. J. Sung, and T. A. Zaki, Inner-Outer Interactions of Large-Scale Structures in Turbulent Channel Flow, J. Fluid Mech. 790, 128 (2016).

[58] S. Fauve and A. Libchaber, Rayleigh-Benard Experiment in a Low Prandtl Number Fluid, Mercury, in Chaos and Order in Nature, Springer Series in Synergetics, edited by $\mathrm{H}$. Haken (Springer, Berlin, 1981), pp. 25-35. 
[59] G. Ahlers, S. Grossmann, and D. Lohse, Heat Transfer and Large Scale Dynamics in Turbulent Rayleigh-Bénard Convection, Rev. Mod. Phys. 81, 503 (2009).

[60] J. Lorat, A. Pouquet, and U. Frisch, Fully Developed MHD Turbulence Near Critical Magnetic Reynolds Number, J. Fluid Mech. 104, 419 (1981).

[61] A. Alexakis, P. D. Mininni, and A. Pouquet, Shell-to-Shell Energy Transfer in Magnetohydrodynamics. I. Steady State Turbulence, Phys. Rev. E 72, 046301 (2005).

[62] A. Azouani and E. S. Titi, Feedback Control of Nonlinear Dissipative Systems by Finite Determining Parameters-A Reaction-Diffusion Paradigm, arXiv:1301.6992.

[63] A. Azouani, E. Olson, and E.S. Titi, Continuous Data Assimilation Using General Interpolant Observables, J. Nonlinear Sci. 24, 277 (2014).

[64] S. Chen and R. H. Kraichnan, Sweeping Decorrelation in Isotropic Turbulence, Phys. Fluids A 1, 2019 (1989).

[65] P. C. di Leoni, P. J. Cobelli, P. D. Mininni, P. Dmitruk, and W. H. Matthaeus, Quantification of the Strength of Inertial Waves in a Rotating Turbulent Flow, Phys. Fluids 26, 035106 (2014).
[66] P. C. di Leoni, P. J. Cobelli, and P. D. Mininni, The SpatioTemporal Spectrum of Turbulent Flows, Eur. Phys. J. E 38, 136 (2015).

[67] A. Sen, P. D. Mininni, D. Rosenberg, and A. Pouquet, Anisotropy and Nonuniversality in Scaling Laws of the Large-Scale Energy Spectrum in Rotating Turbulence, Phys. Rev. E 86, 036319 (2012).

[68] A. Campagne, B. Gallet, F. Moisy, and P.-P. Cortet, Disentangling Inertial Waves from Eddy Turbulence in a Forced Rotating-Turbulence Experiment, Phys. Rev. E 91, 043016 (2015).

[69] L. Biferale, F. Bonaccorso, I. M. Mazzitelli, M. A. T. van Hinsberg, A. S. Lanotte, S. Musacchio, P. Perlekar, and F. Toschi, Coherent Structures and Extreme Events in Rotating Multiphase Turbulent Flows, Phys. Rev. X 6, 041036 (2016).

[70] K. Ide, L. Kuznetsov, and C. K. R. T. Jone, Lagrangian Data Assimilation for Point Vortex Systems, J. Turbul. 3, N53 (2002). 